\title{
Preparedness and Perceived Resilience in a Pandemic: U.S. Cancer Patients during COVID-19
}

\author{
Aldara Henderson, Huaibo Xin* \\ Department of Applied Health, School of Education, Health \& Human Behavior, \\ Southern Illinois University Edwardsville, Illinois, USA
}

Received October 8, 2021; Revised November 18, 2021; Accepted December 13, 2021

\begin{abstract}
Cite This Paper in the following Citation Styles
(a): [1] Aldara Henderson, Huaibo Xin, "Preparedness and Perceived Resilience in a Pandemic: U.S. Cancer Patients during COVID-19," Universal Journal of Public Health, Vol. 10, No. 1, pp. 107 - 115, 2022. DOI: 10.13189/ujph.2022.100112.
\end{abstract}

(b): Aldara Henderson, Huaibo Xin (2022). Preparedness and Perceived Resilience in a Pandemic: U.S. Cancer Patients during COVID-19. Universal Journal of Public Health, 10(1), 107 - 115. DOI: 10.13189/ujph.2022.100112.

Copyright $\bigcirc 2022$ by authors, all rights reserved. Authors agree that this article remains permanently open access under the terms of the Creative Commons Attribution License 4.0 International License

\begin{abstract}
The purpose of this study is to examine U.S. cancer patients' preparedness for a disease outbreak like COVID-19 and understand cancer patients' perceived resilience, and how their preparedness has affected their resilience. It's a cross-sectional study with a national sample of 317 participants. Participants completed an anonymous online survey that consisted of 42 questions on a 5-point Likert scale, addressing different levels of preparedness during a pandemic, and overall individual resilience and COVID-19 resilience. Data were analyzed quantitatively. Results suggested $72 \%$ of the participants somewhat/strongly agreed, individually, they were prepared to respond to a health pandemic. Approximately, $84 \%$ of the participants felt their households had the knowledge to respond to a pandemic. Participants felt their oncologists had enough training $(71 \%)$ but not enough knowledge to respond to a pandemic (58\%). Only $43 \%$ felt healthcare institutions had enough supplies for a health pandemic. Participants somewhat/strongly agreed they were able to recognize thoughts and situations could make them feel stressed or upset about COVID-19 (85\%) and practice their coping skills effectively. There were significant correlations between different levels of preparedness and resilience. Public health preparedness interventions/guidelines tailored towards cancer patients are needed to increase their resilience to a global pandemic.
\end{abstract}

Keywords Disaster Preparedness, Resilience, Cancer, COVID-19

\section{Introduction}

Disaster preparedness constitutes the steps taken to prepare for a disaster and lessen the effects once the disaster occurs [1]. According to the Department of Homeland Security (DHS), it also includes planning for a disaster, training volunteers, practicing for a disaster, evaluating the plans and exercises, and updating the plan as necessary [2]. The Federal Emergency Management Agency (FEMA) identifies that disasters can be meteorological, geological, or biological with minimal to major damage [3]. The American Red Cross (ARC) recommends an emergency kit, a plan, and staying informed on what is going on as a basic template for disaster readiness preparation [4]. The World Health Organization (WHO) published a strategic planning guide for pandemic preparedness in 2005 as a result of the Avian influenza pandemic, which discussed ways to prevent transmission of infections and the importance of anti-viral drugs and vaccines to reduce the number of deaths. It also included the importance of reserving antivirals for those most at risk of being infected and dying of the infection [5]. In reflections on the preparedness during the H1N1 influenza pandemic of 2009 , identifying that while advances in science and medical knowledge have increased since past pandemics, it does not prepare the world for future pandemics. Instead, preparation of treatment protocols and international agreements regarding finances and a supply of public health experts will help mitigate the effects of a global 
pandemic [6].

The National Cancer Institute recommends the following tips for cancer patients in case of a public health emergency: formulate a plan with your healthcare provider and discuss ways to continue communication, make a plan with family and friends using information from government websites, and to create a personal plan and collect all disease-related information including information about cancer, medications, information about clinical trial enrollment, insurance cards and other important paperwork [7]. The National Foundation for the Centers for Disease Control and Prevention (CDC Foundation), an independent nonprofit organization created by Congress to raise money to support the CDC research, recommends patients communicate with their oncologists to set up a plan, not to delay treatments unless ordered by treating physicians, to wash hands to prevent infection, follow social distancing guidelines, and to always wear a mask in public [8]. In one study of COVID-19 knowledge, preparedness, and other factors among breast cancer patients in Indonesia, results showed statistical correlations between COVID-19 awareness and preparedness, COVID-19 knowledge and preparedness, and preparedness with related disaster response behaviors [9].

Resilience is the ability to successfully adapt, in the face of considerable difficulties, such as a large amount of stress, trauma, illness, and tragedy [10]. Resiliency may represent a fixed trait (i.e. a person is naturally resilient or not); however, in reality, it exists within a more fluid state with differing degrees depending on the situation [11]. There are personal factors, such as personality traits, self-esteem, and extroversion, that are a source of resilience. Biological factors, such as genetics and environmental factors including community support, are also other sources of resilience [12]. Multiple studies on resilience and cancer patients have revealed those who have been diagnosed with cancer and have high levels of resilience and have improved overall quality of life versus those with lower levels of resilience [13-17]. Resilience is a protective factor in metastatic cancer patients [13]. One study with breast cancer patients and survivors, discussed how cancer patients are individuals with a goal that is disrupted by cancer and continuing to fulfill that goal, or accessing the individual's resilience, leads to an improved quality of life of cancer survivors [14].With patients receiving genetic testing for familial colorectal cancer, those who are most hopeful are also most resilient, leading researchers to conclude that inspiring hope may lead to more resilient patients and improved quality of life [15]. In metastatic colorectal cancer patients, a strong correlation was found between resiliency and hope, and those patients with lower resilience and less hope tested higher for suffering [16]. In 178 cancer patients with a stage II-IV cancer diagnosis, researchers found a moderate to strong correlation between resilience and the active coping strategies that improve the quality of life for cancer patients. They concluded that a higher perception of quality of life and increased resilience improved a patient's long-term prognosis and can help increase survival rates. Overall resilience can increase by screening baseline resilience levels in patients and providing therapeutic programs [17].

The importance of resilience during the COVID-19 pandemic is also observed across multiple studies. A recently published study concluded that resilience among patients with mild COVID-19 symptoms is a protective factor for depression and anxiety [18]. Having a greater feeling of social connectedness increases resiliency and decreases anxiety and fatigue that is felt among the most anxious people in a pandemic [19]. One study found that although loneliness is a concern for some patients during the COVID-19 pandemic, others enjoyed the slowing down of life and being quarantined with family members and have become more self-reliant, a seemingly negative situation made into a positive situation [20]. High levels of resiliency are also a predictor of COVID-19 induced anxiety, with those with lower levels of resiliency within the general population predicting higher rates of anxiety [21]. Sarcoma patients' experiences and quality of life were explored during the height of the COVID-19 pandemic. More than $70 \%$ of the patients felt the quality of care, which also included telehealth visits, did not diminish during the lockdown. However, patients with lower resilience had a significant higher rate of COVID-19 related worry [22]. The purpose of the current study is to examine US cancer patients' preparedness for a disease outbreak (e.g., COVID-19); understand cancer patients' resilience, the ability to adapt to an adverse situation like COVID-19, and how their emergency preparedness has affected their resilience.

\section{Materials and Methods}

\subsection{Subjects}

Participant recruitment utilized snowball sampling and criteria sampling. In this study, inclusion criteria included participants between the ages of 18-89 who live in the United States, have had a cancer diagnosis, and consented to participate in the survey. Emails with a survey link were shared through personal communications with oncologists across the country. The link was also shared on the Cancer Survivors Network internet page, which is sponsored by the American Cancer Society. Furthermore, participants were recruited from multiple active cancer and survivor-ship groups available on social media (e.g. Facebook Breast Cancer and Sarcoma support groups). G*Power was used to estimate sample size.

\subsection{Measures}

Participants completed a 42-question anonymous online 
survey. The survey included four sets of questions measuring demographics, preparedness in a pandemic, general resilience, and resilience during the COVID-19 pandemic. (1) Demographics included age, sex, race, type of cancer, the staging of cancer (0-5), and whether the participants had active cancer or had no evidence of disease (NED). (2) Preparedness questions for a public health emergency (e.g., COVID-19) were adopted from a published survey and modified to specifically address cancer patients' preparedness [23]. The response options used a 5-point Likert scale. These statement questions were further expanded to evaluate individual preparedness, household preparedness, and institutional preparedness. Examples of statements for individual preparedness included: "I find myself as a cancer patient prepared to respond to emergencies caused by a pandemic (For example: COVID-19, MERS, Bird Flu etc.)," "I find that I have enough supplies of food, medicine, etc. during a pandemic," and "I find that I have the necessary protective equipment during a pandemic." These statements were reworded to address household preparedness. Institutional preparedness statements included: "I believe that my oncologist has enough knowledge to respond during a pandemic (For example: COVID-19, MERS, Bird Flu etc.)," "I believe that my oncologist has enough training to respond during a pandemic," and "I believe that my local cancer center has enough supplies to respond during a pandemic" [23]. (3) The Brief Resilience Coping Scale (BRCS) was adopted to examine participants' overall/general resilience [24]. The BRCS consists of four statements and uses a 5-point Likert scale and has demonstrated validity with multiple population groups (German population, medical students, and older adults) and for diseases such as systematic lupus [25]. Participants with scores between 4-13 had low resilience, 14-16 had medium resilience, and 17-20 were considered highly resilient [24]. (4)The specific questions addressing the ability to handle a particular stressor/trauma were adopted from a published survey and modified to explore the resilience of cancer patients during the COVID-19 pandemic using a 5-point Likert scale with statements such as: "Since the breakout of the COVID-19 pandemic, I am able to recognize thoughts and situations that make me feel stressed or upset about COVID-19," and "I am able to practice relaxation (e.g., deep breathing, meditation) when feeling stress about COVID-19" [26]. The complete survey was reviewed by colleagues in public health, cancer patients, and an oncologist for its reliability and validity.

\subsection{Procedure}

The data were obtained through a cross-sectional study using an online survey to assess the preparedness and resilience of cancer patients in the U.S. during a global health crisis. The survey remained open from January 4th,
2021 until March 2nd, 2021. The purpose of the study was detailed, and consent was obtained from each participant at the beginning of the survey. The survey took no more than 15 minutes to complete. This study was approved by the university's Institutional Review Board \#930.

\subsection{Analysis}

Descriptive statistics were performed using IBM SPSS version 26 (IBM). Any missing responses were not included in the analysis. Further stratification of responses was conducted to distinguish between those in active treatment and those who identified as survivors to see if there was a difference in resiliency traits and preparedness in both groups. The final analytic sample included 317 participants. Descriptive statistics were used to conduct frequencies and percentages. Analysis was conducted using Independent Samples t-tests to identify any differences in scores between demographic variables (employed and others, before COVID-19 cancer diagnosis and diagnosed during the pandemic, active cancer patients and survivors, and breast cancer patients and those with other cancer diagnosis) to the various preparedness (individual, household, institutional, and overall) and resilience (overall resilience and COVID-19 resilience) scores. One-way Analysis of Variance (ANOVA) with post hoc testing for multiple comparisons within the groups, using 0.05 as the statistically significant level, was conducted to compare means of various groups (the three different levels of resilience from the BRCS and the five stages of cancer classifications) to the various preparedness variables and resilience during COVID-19. In addition, correlation analysis was conducted to identify the strength and direction of the relationship between preparedness and resilience.

\section{Results}

A total of 317 cancer patients participated in the study. Among the reported (Table 1), those between the ages of $37-55$ made up $47 \%$ of the participants, whereas $75-89$ year old participants only contributed $3 \%$ of the participants. The majority $(90 \%)$ of the participants were identified as female. Furthermore, over $90 \%$ were identified as White (91\%) and of non-Hispanic of Latinx origin (94\%). Approximately $77 \%$ had a bachelor's degree and attended graduate school. Almost $62 \%$ stated they were employed and the largest salary range $(31 \%)$ was between \$60,000-99,999. Regionally, participants were almost evenly split between the Midwest (31\%), the South (31\%), and the Western US (28.3\%). The Northeast had only $9 \%$ of the participants. Approximately $25 \%$ of the participants were in active cancer treatment and $75 \%$ were post treatment. With only $7 \%$ diagnosed at stage 0 , almost $75 \%$ of participants said they were diagnosed between 
stages one and three and $18 \%$ were diagnosed at stage 4 . About $38 \%$ of the participants were breast cancer patients and $62 \%$ were a combination of the different cancers ranging from Sarcoma to prostate cancer. Additionally,
$85 \%$ received their cancer diagnosis before the start of the COVID-19 pandemic, with only $15 \%$ receiving their diagnosis during the pandemic.

Table 1. Participant's Demographics

\begin{tabular}{|c|c|}
\hline Demographics & Number (Percentage) \\
\hline $\begin{array}{c}\text { Age } \\
18-36 \\
37-55 \\
56-74 \\
75-89 \\
\text { Total }\end{array}$ & $\begin{array}{c}53(19.1) \\
131(47.1) \\
87(31.3) \\
7(2.5) \\
278\end{array}$ \\
\hline $\begin{array}{c}\text { Gender } \\
\text { Male } \\
\text { Female } \\
\text { Total }\end{array}$ & $\begin{array}{c}29(9.8) \\
268(90.2) \\
297\end{array}$ \\
\hline $\begin{array}{c}\text { Race } \\
\text { American Indian or Alaska Native } \\
\text { Asian } \\
\text { Black or African American } \\
\text { Native Hawaiian or Pacific Islander } \\
\text { White } \\
\text { Other } \\
\text { Total }\end{array}$ & $\begin{array}{c}1(.3) \\
8(2.7) \\
4(1.4) \\
1(.3) \\
269(91.2) \\
12(4.1) \\
295\end{array}$ \\
\hline $\begin{array}{c}\text { Ethnicity } \\
\text { Hispanic or Latino } \\
\text { Not Hispanic or Latino } \\
\text { Total }\end{array}$ & $\begin{array}{c}16(5.8) \\
259(94.2) \\
275\end{array}$ \\
\hline $\begin{array}{c}\text { Education Level } \\
\text { High School through Associates } \\
\text { Bachelor's Degree } \\
\text { Graduate School } \\
\text { Total }\end{array}$ & $\begin{array}{c}87(29.2) \\
101(33.9) \\
110(36.9) \\
298\end{array}$ \\
\hline $\begin{array}{c}\text { Employment Status } \\
\text { Employed } \\
\text { Other } \\
\text { Total }\end{array}$ & $\begin{array}{c}180(61.2) \\
114(38.8) \\
294\end{array}$ \\
\hline $\begin{array}{c}\text { Household Income (US Dollars) } \\
0-59,999 \\
60,000-99,999 \\
100,000-149,000 \\
\text { More than } 150,000 \\
\text { Total }\end{array}$ & $\begin{array}{l}77(27.1) \\
87(30.6) \\
70(24.6) \\
50(17.6) \\
284\end{array}$ \\
\hline $\begin{array}{c}\text { Health Insurance } \\
\text { Medicaid } \\
\text { Medicare } \\
\text { Employer Sponsored Disability } \\
\text { Private of Group Health Insurance } \\
\text { National Health Insurance } \\
\text { Veterans Affairs/Military } \\
\text { No Insurance/Self-pay } \\
\text { Unknown } \\
\text { Other } \\
\text { Total }\end{array}$ & $\begin{array}{c}11(3.7) \\
51(17.1) \\
31(10.4) \\
170(57.0) \\
5(1.7) \\
12(4.0) \\
7(2.3) \\
1(.3) \\
10(3.4) \\
298\end{array}$ \\
\hline $\begin{array}{c}\text { Location (By region) } \\
\text { Midwest } \\
\text { Northeast } \\
\text { South } \\
\text { West } \\
\text { Total }\end{array}$ & $\begin{array}{c}90(31.3) \\
27(9.4) \\
89(30.9) \\
82(28.3) \\
288\end{array}$ \\
\hline
\end{tabular}


Table 2. Frequencies and Percentages of Statements in the Brief Resilience Coping Scale (BRCS)

\begin{tabular}{|l|c|c|c|c|c|c|}
\hline & $\begin{array}{c}\text { Does not describe } \\
\text { me at all }\end{array}$ & $\begin{array}{c}\text { Does not } \\
\text { describe me }\end{array}$ & Neutral & Describes me & $\begin{array}{c}\text { Describes me } \\
\text { well }\end{array}$ & N \\
\hline $\begin{array}{l}\text { I look for creative ways to } \\
\text { alter difficult situations. }\end{array}$ & $2(0.8)$ & $11(4.6)$ & $28(11.8)$ & $131(55.0)$ & $66(27.7)$ & 238 \\
\hline $\begin{array}{l}\text { Regardless of what happens } \\
\text { to me, I believe I can control } \\
\text { my reactions to it. }\end{array}$ & $4(1.7)$ & $27(11.3)$ & $35(14.7)$ & $127(53.4)$ & $45(18.9)$ & 238 \\
\hline $\begin{array}{l}\text { I believe I can grow in } \\
\text { positive ways by dealing with } \\
\text { difficult situations. }\end{array}$ & $0(0.0)$ & $3(1.3)$ & $33(13.9)$ & $122(51.3)$ & $80(33.6)$ & 238 \\
\hline $\begin{array}{l}\text { I actively look for ways to } \\
\text { replace the losses I encounter } \\
\text { in life. }\end{array}$ & $3(1.3)$ & $30(12.6)$ & $67(28.2)$ & $103(43.3)$ & $35(14.7)$ & 238 \\
\hline
\end{tabular}

The frequencies and percentages of Individual, Household, and Institutional Preparedness were also illustrated. For individual preparedness, more than $60 \%$ of the survey participants somewhat and strongly agreed with all the statements. For example, $69 \%$ of participants felt they had enough supplies of food and medicine during a pandemic. Almost $80 \%$ felt they had enough knowledge to respond to a pandemic. However, less than $60 \%$ somewhat and strongly agreed they had the necessary protective equipment during a pandemic. For household preparedness, every statement had at least $70 \%$ of the participants somewhat and strongly agreeing. For example, $72 \%$ of participants both somewhat and strongly agreed they had enough supplies in their household to respond to a pandemic. Similar to individual preparedness, the greatest percentage of participants $(84 \%)$ both somewhat and strongly agreed with the statement acknowledging they felt there was enough knowledge within their household to respond during a pandemic. Additionally, $75 \%$ of participants felt they had effective plans within their household to respond during a pandemic. For institutional preparedness, most statements had at least 50\% of survey participants who somewhat and strongly agreed with the statements. Participants agreed $(71 \%)$ that their oncologist had enough training to respond during a pandemic. Also, $68 \%$ felt their healthcare institutions have enough knowledge to respond to a pandemic. However, only $43 \%$ agreed that health care institutions had enough supplies to respond to a pandemic.

The BRCS (Table 2) indicated that about $83 \%$ of the participants generally believed that their practice could alter significant adverse situations and believed they could grow in positive ways in difficult situations (85\%). No one felt that growing in positive ways by dealing with difficult situations did not describe them at all. Only 58\% of participants looked for ways to replace the losses encountered in life. In relation to COVID-19, participants also somewhat/strongly agreed they were able to recognize thoughts and situations that make them feel stressed or upset about COVID-19 (85\%) and to seek information and plan accordingly (83\%). Also, $67 \%$ of participants felt they could give themselves the caring and tenderness needed during COVID-19. However, only $62 \%$ felt they were able to practice relaxation skills when feeling stressed about the COVID-19 pandemic.

Multiple independent samples $\mathrm{t}$-tests were conducted to compare preparedness and resilience scores to several different variables such as employed and others (unemployed, unable to work, homemaker, student, and retired); before COVID-19 cancer diagnosis and diagnosed during the pandemic; active cancer patients and survivors; and breast cancer patients and other cancer diagnosis. The t-tests yielded no significant differences in any of the scores.

In addition, One-way ANOVA compared means of individual preparedness total, household preparedness total, institutional preparedness total, overall preparedness, and resilience during COVID-19 among three different levels of resilience (low, medium and high) measured by the BRCS, followed by Tukey HSD Post Hoc Multiple Comparisons. Significant differences were found. Those that scored in the low resilience range had significantly less individual preparedness total scores than those with medium and high resilience $(\mathrm{p}=.02 ; \mathrm{p}=.00)$. There was a significant difference between those with medium resilience and high resilience regarding individual preparedness $(\mathrm{p}=.02)$. Regarding household preparedness, those with low resilience felt their household were not as prepared as those with medium resilience and high resilience $(p=.00 ; p=.00)$. Again, there was also a significant difference in household preparedness between medium and high resilience groups $(p=.01)$. Institutional preparedness found that low resilient participants felt their institutions were not as prepared as those with medium resilience or high resilience $(p=.03 ; p=.00)$. In overall preparedness, low resilience participants did not feel as prepared overall to a pandemic as those with medium resilience and high resilience $(p=.00 ; p=.00)$. Significant differences were also found between medium and high resilient individuals in overall preparedness $(p=.01)$. Low resilience participants felt significantly worse compared to those with medium $(p=.00)$ and high resilience $(p=.00)$ in showing resilience during COVID-19. There was also a significant difference between medium and high resilience 
$(\mathrm{p}=.00)$ in exhibiting resilience during COVID-19.

Significant differences were also identified after comparing the means of individual preparedness total, household preparedness total, institutional preparedness total, overall preparedness, and resilience during covid-19 among the five stages of cancer classification. Those diagnosed with stage one cancer felt their individual preparedness was significantly greater than those with stage 2 cancer $(\mathrm{p}=.01)$. Within household preparedness, those considered stage one felt significantly more prepared than those with stage 2 and stage $4(p=.04$; $\mathrm{p}=.03$ ). In overall preparedness, those diagnosed with stage 1 cancer identified themselves as significantly more prepared than those with stage 2 cancer $(\mathrm{p}=.02)$.

There were multiple positive relationships between overall resilience, measured by the BRCS, and individual preparedness $(\mathrm{r}=.28, \mathrm{p}=.00)$, household preparedness $(\mathrm{r}=.32, \mathrm{p}=.00)$, institutional preparedness $(\mathrm{r}=.24, \mathrm{p}=.00)$, and overall preparedness $(\mathrm{r}=.32, \mathrm{p}=.00)$ with higher levels of overall resilience associated with higher levels of preparedness. Moreover, there were multiple positive and significant relationships between COVID-19 resilience and individual preparedness $(\mathrm{r}=.34, \mathrm{p}=.00)$, household preparedness $(\mathrm{r}=.38, \mathrm{p}=.00)$, institutional preparedness $(\mathrm{r}=.20, \mathrm{p}=.00)$, and overall preparedness $(\mathrm{r}=.34, \mathrm{p}=.00)$ with higher levels of resilience associated with higher levels of preparedness.

\section{Discussion}

The current study actively recruited participants from a variety of web-based support networks, but only a quarter of the participants were in active treatment. This study also follows the trend presented in different studies of more female and highly educated individuals participating in online surveys [27]. The vast majority $(90 \%)$ of the participants were women. Additionally, almost two-thirds of the participants were educated with at least a bachelor's degree or higher, and three-quarters of the participants made the average gross income in the United States or higher. Furthermore, 91\% were identified as white.

The greatest concern in preparedness in this study was in the participants' confidence in institutional preparedness. Although participants were at least somewhat confident in the knowledge and training of their oncologists to handle a pandemic like COVID-19, along with cancer centers having the plan and supplies to deal with a pandemic, they were much less confident in health care institutions' knowledge and supplies during a health pandemic. Recommendations for a standard of care specifically for cancer patients are necessary during a crisis, whether a natural disasters or pandemic, with a defined set of terms and universal treatment protocols for cancer patients [28]. For example, distinguishing between what is considered an elective procedure versus one that is essential, and also how to create a uniform and ethical triage system for the distribution of resources. This is beneficial in a pandemic as it provides guidelines across all oncological governing boards and medical facilities. Moreover, discussion of telehealth procedures is critical during a pandemic, specifically for reimbursement of medical fees and the necessity to approve oncology clinicians to receive licenses to practice in other states, whether through travel or providing telehealth care [28]. The Interstate Medical License Compact Commission (IMLCC), a medical governing body, has 32 states that allow medical professionals to practice medicine, in person or telehealth visits, within those states [29]. Having all 50 states and US territories as a member of this organization would allow for additional availability of services during disasters or pandemics, especially in hardest hit areas. Furthermore, researchers have recommended for medical institutions during a pandemic the importance of testing health policies and crisis development, identifying facilities or academic centers that can identify and create accurate tests of the pathogen causing a pandemic, increasing facilities and beds to help accommodate a health crisis, using makeshift hospitals as the primary treatment sites of pandemic patients allowing hospitals to continue to treat typical patients, and streamlining practice procedures for treatments [30]. Additionally, contingency plan development, including a focus on end of life and palliative care support along with the need for improved web-based healthcare access is necessary [30]. Although most pandemic preparedness information and lessons learned during COVID-19 are general in nature, the recommendations provided can be tailored towards cancer patients and may help in the planning and health policies geared towards cancer patients during future pandemics.

Although COVID-19 is a fluid situation and more information is becoming available addressing cancer patients' needs during global health pandemics, it is also possible to extrapolate information about cancer patients in other types of disasters to prepare for future health pandemics. For example, in the 2011 nuclear disaster in Fukushima Japan, social isolation, inadequate access to diagnostic treatment, and fear of exposure led to delayed diagnosis in breast cancer patients [31]. During Hurricane Katrina, cancer care was severely disrupted because of the destruction of buildings and treatment centers. Records were lost, treatments were delayed, and supply chains of medical supplies were disrupted. However, multiple organizations reached out into the community to help those affected along with creating more robust electronic record databases to help continue research and medical trials [32]. A systematic review to identify critically ill patients, including cancer patients, in disasters and pandemics between 1985 and 2013, found very little for disaster preparedness of this vulnerable population. Therefore, they presented their own recommendations for 
critically ill patients, which included the following: disaster planning for critical care populations (including cancer patients), disaster preparedness plans formulated with their primary care providers (e.g., oncologists for cancer patients), and pre-identifying patients who will require more help during a disaster [33].

Regarding participants' resilience, per the BRCS, almost $82 \%$ of the study participants scored in the medium and high range of resilience. It is possible that cancer survivors may present a higher level of resilience compared to the general population, which can be further studied. At least $80 \%$ of participants felt that looking for creative ways to alter difficult situations and growing in positive ways by dealing with difficult situations described them. But fewer participants felt they could control their reactions to experiences in life or replace the losses encountered in life. In analyzing COVID-19 resilience, at least $70 \%$ of participants felt they were able to recognize thoughts and stress related to COVID-19, seek information about COVID-19 and reexamine negative thoughts and gain new perspectives; a smaller percentage $(60 \%)$ of participants had more difficulty practicing relaxation and self-care. The Mayo Clinic recommends the following ways to build resilience in the general population that can be adapted for cancer patients: by building connections and fostering relationships with family and friends and within the community, making their days meaningful and accomplishing tasks, creating a journal describing coping mechanisms used during bad times to help in the future, looking towards the future and learning to adapt to changes, practicing stress management techniques, and being proactive with problems and not ignoring them [34]. In a model of resilience geared towards cancer patients and survivorship, focus should be on both the initial diagnosis, the acute trauma, and also the long-term trauma, which includes undergoing treatments and post treatment survivorship [35]. Another study further describes initial attributes for resilience such as personality, intelligence, and the ability to have good self-control, along with demographic factors such as age, sex, and socioeconomic status, and even included a genetic component to resilience. They also describe the process of coping from diagnosis through treatments, and included types of interventions that may be beneficial, such as being supportive to those survivors who are adaptive, and teaching relaxation techniques to highly anxious patients [35]. Additionally, patients with lower resilience levels also have the highest number of unmet needs in newly diagnosed cancer patients and by encouraging the patients' resiliency, it may reduce the number of unmet needs [36].

Prior to this current study, there are no existing studies examining the association of preparedness and resilience of cancer patients during the COVID-19 pandemic. Notably, the present study did find a significant relationship between preparedness and resilience. Given the relationship between levels of preparedness and resilience, it is important that cancer patients are provided with information in case of future disasters and pandemics. Increased uncertainty among cancer patients leads to lower levels of resilience [37] Providing disaster preparedness checklists in welcome packets at cancer centers or detailed information online can provide cancer patients the information they need to be prepared. Those having a strong preparedness belief were more likely to have a kit and a plan in case of an emergency.38 Encouraging disaster preparedness and lowering uncertainty of cancer patients with communication of facts and plans could increase resilience among cancer patients.

\section{Conclusion}

Public health preparedness interventions and guidelines tailored towards cancer patients are needed to increase their resilience to a public health emergency. Research has shown, including this study, that higher levels of resilience are an integral component for weathering a future pandemic [18-21, 37], and being able to use coping and stress reducing mechanisms successfully in a pandemic may help with reducing mental issues associated with a pandemic [39-41]. Integrating and continuing resilience building therapies for cancer patients, along with providing preparedness information and guidelines for cancer patients before and during a disaster, become essential to this vulnerable population.

\section{REFERENCES}

[1] International Federation of Red Cross and Red Crescent Societies, "Disaster preparedness." https://media.ifrc.org/if rc/what-we-do/disaster-and-crisis-management/disaster-pre paredness/

[2] Department of Homeland Security, "Plan and Prepare for Disasters," 2020. https://www.dhs.gov/plan-and-prepare-di sasters

[3] Federal Emergency Management Agency, "Glossary." https://training.fema.gov/programs/emischool/el361 toolkit/ glossary.htm\#D

[4] Red Cross, "How to prepare for emergencies." https://www.redcross.org/get-help/how-to-prepare-for-eme rgencies.html

[5] World Health Organization, "Recommended strategic actions: Responding to the avian influenza pandemic threat," 2020. https://www.who.int/csr/resources/publications/influ enza/WHO_CDS_CSR_GIP_05_8-EN.pdf

[6] Fineberg H. V., "Pandemic Preparedness and Response: Lessons from the H1N1 Influenza of 2009," New England Journal of Medicine, vol. 370, no, 14, pp. 1335-1342, 2004. 


\section{DOI: $10.1056 /$ nejmra1208802}

[7] National Cancer Institute, "Emergency resources for the cancer community," 2021. https://www.cancer.gov/contact /emergency-preparedness

[8] Centers for Disease Control and Prevention, "Prevent Cancer Infections." https://www.cdc.gov/hai/pdfs/bicapp/c dc_pocketguide_final_508compliant.pdf

[9] Nindrea R. D., Sari N. P., Harahap W. A., et al., "Survey data of COVID-19 awareness, knowledge, preparedness and related behaviors among breast cancer patients in Indonesia," Data in Brief, vol. 32, pp. 106145-106150, 2020. DOI: $10.1016 /$ j.dib.2020.106145

[10] American Psychological Association, "Building your resilience," 2012. https://www.apa.org/topics/resilience

[11] Southwick S. M., Bonanno G. A., Masten A. S., Panter-Brick C., Yehuda R., "Resilience definitions, theory, and challenges: Interdisciplinary perspectives," European Journal of Psychotraumatology, vol. 5. no. 1, pp. 25338-25351, 2014. DOI: 10.3402/ejpt.v5.25338

[12] Herrman H., Stewart D. E., Diaz-Granados N., Berger E. L., Jackson B., Yuen T., "What is resilience?" Canadian Journal of Psychiatry, vol. 56, no. 5, pp. 258-265, 2011. DOI: $10.1177 / 070674371105600504$

[13] Min J. A., Yoon S., Lee C. U., et al., "Psychological resilience contributes to low emotional distress in cancer patients," Supportive Care in Cancer, vol. 21, no. 9, pp. 2469-2476, 2013. DOI: 10.1007/s00520-013-1807-6

[14] Carver C. S., "Enhancing adaptation during treatment and the role of individual differences," International Journal of the American Cancer Society, vol. 104, no. S11, pp. 2602-2607, 2005. DOI: 10.1002/cncr.21247

[15] Ho S. M., Ho J. W., Bonanno G. A., Chu A. T., Chan E. M., "Hopefulness predicts resilience after hereditary colorectal cancer genetic testing: A prospective outcome trajectories study," BMC Cancer, vol. 10, no. 1, pp. 1-10, 2010. DOI: $10.1186 / 1471-2407-10-279$

[16] Solano J. P. C., da Silva A. G., Soares I. A., Ashmawi H. A., Vieira J. E., "Resilience and hope during advanced disease: A pilot study with metastatic colorectal cancer patients," BMC Palliative Care, vol. 15, no. 1, pp. 1-8, 2016. DOI: 10.1186/s12904-016-0139-y

[17] Popa-Velea O., Diaconescu L., Jidveian Popescu M., Truţescu C., "Resilience and active coping style: Effects on the self-reported quality of life in cancer patients," The International Journal of Psychiatry in Medicine, vol. 52, no. 2, pp. 124-136, 2017. DOI: 10.1177/0091217417720895

[18] Zhang J., Yang Z., Wang X., Li J., Dong L., Wang, F., ... Zhang J., "The relationship between resilience, anxiety and depression among patients with mild symptoms of COVID - 19 in China: A cross - sectional study," Journal of Clinical Nursing, vol. 29, no. 21-22, pp. 4020-4029, 2020. DOI: $10.1111 /$ jocn. 15425

[19] Nitschke J. P., Forbes P. A., Ali N., Cutler J., Apps M. A., Lockwood P. L., Lamm C., "Resilience during uncertainty? Greater social connectedness during COVID - 19 lockdown is associated with reduced distress and fatigue," British Journal of Health Psychology, vol. 26, no. 2, pp. 553-569 2021. DOI: 10.1111/bjhp.12485
[20] Schellekens M. P., van der Lee M. L., "Loneliness and belonging: Exploring experiences with the COVID-19 pandemic in psycho-oncology," Psychooncology, vol. 29, no. 9 , pp. 1399-1401, 2020. DOI: 10.1002/pon.5459.

[21] Kimhi S., Marciano H., Eshel Y., Adini B., "Resilience and demographic characteristics predicting distress during the COVID-19 crisis," Social Science and Medicine, vol. 265, pp. 113389-113394, 2020. DOI: 10.1016/j.socscimed.2020 .113389

[22] Younger E., Smrke A., Lidington E., Farag S., Ingley K., Chopra N., ... Jones R. L., "Health-related quality of life and experiences of sarcoma patients during the COVID-19 pandemic," Cancers, vol. 12, no. 8, pp. 2288-2313, 2020. DOI: $10.3390 /$ cancers 12082288

[23] Cvetković V. M., Nikolić N., Radovanović Nenadić U., Öcal A., Noji E. K., Zečević M., "Preparedness and preventive behaviors for a pandemic disaster caused by COVID-19 in Serbia," International Journal of Environmental Research and Public Health, vol. 17, no. 11, pp. 4124-4146, 2020. DOI: 10.3390/ijerph17114124

[24] Sinclair V. G., Wallston K. A., "The development and psychometric evaluation of the Brief Resilient Coping Scale," Assessment, vol. 11, no. 1, pp. 94-101. DOI: $10.1177 / 1073191103258144$

[25] Kocalevent R. D., Zenger M., Hinz A., Klapp B., Brähler E., "Resilient coping in the general population: Standardization of the brief resilient coping scale (BRCS)," Health and Quality of Life Outcomes, vol. 15, no. 1, pp. 1-8, 2017. DOI: 10.1186/s12955-017-0822-6

[26] Penedo F. J., Cohen L. G., Bower J., Antoni M., "Covid-19: Impact of the pandemic and HRQOL in cancer patients and survivors," 2020. https://www.nlm.nih.gov/dr2/COVID-19 _IMPACT_HRQOL_QUESTIONNAIRE_FINAL.pdf

[27] Smith W. G., "Does gender influence online survey participation? A record-linkage analysis of university faculty online survey response behavior," 2008. http://files.eric.ed.gov/fulltext/ED501717.pdf

[28] Kelkar A. H., Cogle C. R., "Cancer in the time of Coronavirus: A call for Crisis Oncology Standards of care," Healthcare (Basel), vol. 8. no. 3, pp. 214-221, 2020. DOI: $10.3390 /$ healthcare 8030214

[29] Robeznieks A., "Cross-state licensing process now live in 30 states," 2021. https:/www.ama-assn.org/practice-mana gement/digital/cross-state-licensing-process-now-live-30-st ates

[30] Timmis K., Brüssow H., "The COVID-19 pandemic: Some lessons learned about crisis preparedness and management, and the need for international benchmarking to reduce deficits," Environmental Microbiology, vol. 22, no. 6, pp. 1986-1996, 2020. DOI: 10.1111/1462-2920.15029

[31] Ozaki A., Leppold C., Tsubokura M., et al., "Social isolation and cancer management after the 2011 triple disaster in Fukushima, Japan: A case report of breast cancer with patient and provider delay," Medicine (Baltimore), vol. 95, no. 26, pp. e4027-e4030, 2016. DOI: 10.1097/MD.0000000000004027

[32] Twombly R., "Cancer community offers unprecedented support after hurricanes slam US Gulf Coast," Journal of the 
National Cancer Institute, vol. 97, no. 23, pp. 1716-1718, 2005. DOI: $10.1093 /$ jnci/dji439

[33] Dries D., Reed M. J., Kissoon N., Christian M. D., Dichter J. R., Devereaux A. V., Upperman J. S., "Special populations: Care of the critically ill and injured during pandemics and disasters: CHEST consensus statement," Chest, vol. 146, no. 4, pp. e75S-e86S, 2014. DOI: 10.1378/chest.14-0737

[34] Mayo Clinic, "Resilience: Build skills to endure hardship," 2020.https://www.mayoclinic.org/tests-procedures/resilien ce-training/in-depth/resilience/art-20046311

[35] Deshields T. L., Heiland M. F., Kracen A. C., Dua P., "Resilience in adults with cancer: Development of a conceptual model: Resilience in adults with cancer," Psychooncology, vol. 25, no. 1, pp. 11-18, 2016. DOI: 10.1002/pon.3800

[36] Dubey C., De Maria J., Hoeppli C., Betticher D. C., Eicher M., "Resilience and unmet supportive care needs in patients with cancer during early treatment: A descriptive study," European Journal of Oncology Nursing, vol. 19, no. 5, pp. 582-588, 2015. DOI: 10.1016/j.ejon.2015.03.004

[37] Cha K., Kim K., "Impact of uncertainty on resilience in cancer patients," Asian Oncology Nursing, vol. 12, no. 2, pp. 139-146, 2012. DOI: 10.5388/aon.2012.12.2.139
[38] Thomas T. N., Leander-Griffith M., Harp V., Cioffi J. P., "Influences of preparedness knowledge and beliefs on household disaster preparedness," Morbidity and Mortality Weekly Report, vol. 64, no. 35, pp. 965-971, 2015. https://www.cdc.gov/MMWR/preview/mmwrhtml/mm643 $5 \mathrm{a} 2 . \mathrm{htm}$

[39] Karatzias T., Shevlin M., Murphy J., McBride O., Ben Ezra M., Bentall R. P., ... Hyland P., "Posttraumatic stress symptoms and associated comorbidity during the COVID 19 pandemic in Ireland: A population - based study," Journal of Traumatic Stress, vol. 33, no. 4, pp. 365-370, 2020. DOI: $10.1002 /$ jts. 22565

[40] Vazquez C., Valiente C., García F. E., Contreras A., Peinado V., Trucharte A., Bentall R. P., "Post-traumatic growth and stress-related responses during the COVID-19 pandemic in a national representative sample: The role of positive core beliefs about the world and others," Journal of Happiness Studies, vol. 22, pp. 2915-2935, 2021. DOI: $10.1007 / \mathrm{s} 10902-020-00352-3$

[41] Brewin C. R., DePierro J., Pirard P., Vazquez C., Williams R., "Why we need to integrate mental health into pandemic planning," Perspectives in Public Health, vol. 140, no. 6, pp. 309-310, 2020. DOI: 10.1177/1757913920957365 